\title{
Modalité standardisée de divulgation de conflit d'intérêts pour le CJHP
}

par Peter J Zed

$\mathrm{L}$ a divulgation de conflits d'intérêts réels ou potentiels d'auteurs d'articles publiés dans la documentation biomédicale est devenue la norme pour la plupart des revues. La crédibilité des articles publiés dépend, en partie, de la rigueur avec laquelle les conflits sont traités et déclarés. Il y a conflit d'intérêts lorsqu'un auteur a des liens financiers ou personnels qui peuvent influencer de façon inappropriée ou biaiser ses actions. De tels liens peuvent être mineurs ou très importants et la possibilité d'un conflit d'intérêts peut exister indépendamment que l'auteur croit ou non que ce lien affecte son jugement scientifique. Des liens financiers, comme la relation d'emploi, la détention d'actions, la relation de consultant et les honoraires versés à un conférencier ou le remboursement de ses frais de déplacement sont les conflits d'intérêts les plus courants et également ceux qui risquent le plus de miner la crédibilité des auteurs, de la science et de la revue dans laquelle le travail est publié. La divulgation des conflits d'intérêts permet au lecteur d'évaluer la nature des liens entre les auteurs et les entités commerciales qui pourraient avoir des intérêts concurrents quant à l'information présentée dans le manuscrit publié.

Malgré le fait que de nombreuses revues exigent que les auteurs déclarent tout conflit d'intérêts, il n'existe aucun modèle pour assurer l'uniformité des divulgations. Les auteurs peuvent donner des renseignements similaires quant aux conflits d'intérêts dans des formes différentes, ce qui peut porter à confusion à la fois pour l'auteur et le lecteur. De plus, les exigences des différentes revues en ce qui a trait à ces divulgations demandent beaucoup de temps de la part des auteurs. Pour résoudre ce problème, l'International Committee of Medical Journal Editors (ICMJE) a créé un formulaire standardisé de divulgation de conflits d'intérêts qui a été adopté par toutes les revues membres de l'ICMJE', y compris le New England Journal of Medicine, The Lancet, les Annals of Internal Medicine, le BMJ, le Journal of the American Medical
Association et le Journal de l'Association médicale canadienne. Ce processus de divulgation standardisé demande aux auteurs de déclarer quatre types de renseignements selon lesquels pourrait exister un conflit d'intérêts réel ou potentiel : 1) leurs liens avec des entités commerciales leur ayant apporté un soutien pour le travail rapporté dans le manuscrit (en cours d'exécution du travail évalué pour publication), 2) leurs liens avec des entités commerciales pouvant être perçues comme ayant un intérêt dans le domaine général faisant l'objet du manuscrit soumis (dans les 36 mois précédant la soumission), 3) tout lien financier similaire impliquant leur conjoint ou leur conjointe ou leurs enfants de moins de 18 ans et 4) tout lien non financier pouvant être pertinent dans le cadre du manuscrit soumis.

Les membres du Comité de rédaction du JCPH sont d'avis qu'il s'agit d'une importante avancée dans la divulgation des conflits d'intérêts réels ou potentiels, et ceuxci ont par conséquent adopté ce processus de divulgation standardisé ainsi que le formulaire pertinent pour les manuscrits soumis au JCPH. Le formulaire et les instructions pour le remplir sont affichés sur le site Web du JCPH (www.cjhp-online.ca/pages/files/ICMJE_Disclosure_ Form.pdf). À compter de maintenant, tous les auteurs devront remplir et remettre ce formulaire en même temps qu'ils soumettent leur manuscrit au JCPH. Bien que le formulaire puisse sembler intimidant à première vue, l'auteur peut facilement le télécharger puis y faire la mise à jour de ses renseignements chaque fois qu'il soumet un manuscrit à une revue. L'adoption de ce formulaire standard par le JCPH, ainsi que par les membres de l'ICMJE et d'autres revues participantes, éliminera le besoin de reformater les formulaires de divulgation de conflits d'intérêts particuliers à chaque revue, ce qui permettra également aux auteurs de gagner du temps. De plus, chaque membre du Comité de rédaction du JCPH devra remplir ce formulaire 
annuellement. Nous sommes reconnaissants du soutien qu'apportent nos auteurs au JCPH en soumettant leurs manuscrits et nous sommes sensibles aux besoins de notre lectorat. Nous croyons que cette modalité standardisée de divulgation d'intérêts concurrents est dans le meilleur intérêt de toutes les parties prenantes du JCPH.

[Traduction par l'éditeur]

\section{Reference}

1. Drazen JM, Van Der Weyden MB, Sahni P, Rosenberg J, Marusic A, Laine C, et al. Uniform format for disclosure of competing interests in ICEMJ journals [éditorial]. International Committee of Medical Journal Editors; 2009. Publié au www.icmje.org/format.pdf. Consulté le 12 avril 2010.
Peter J Zed, B. Sc., B. Sc. Pharm., ACPR, Pharm. D., FCSHP, est coordonnateur clinique, Département de pharmacie, et spécialiste de la pharmacothérapie, Médecine d'urgence, au Queen Elizabeth I Health Sciences Centre, et professeur agrégé, Collège de pharmacie et Département de médecine d'urgence, Université Dalhousie, Halifax, Nouvelle-Écosse. Il est également rédacteur adjoint du CHHP.

\section{Address correspondence to:}

Dr Peter J Zed

Department of Pharmacy

Halifax Infirmary

Queen Elizabeth II Health Sciences Centre

1796 Summer Street

Halifax NS B3H 3 A7

courriel : peter.zed@dal.ca

\section{Canadian Society of Hospital Pharmacists}

Société canadienne des pharmaciens d'hôpitaux

\section{CSHP's Online Continuing Education Program}

The Canadian Society of Hospital Pharmacists (CSHP) is proud to present its online education program with courses accredited by the Canadian Council on Continuing Education in Pharmacy (CCCEP). The online education program will help CSHP to deliver innovative educational programs to health care professionals from across the country.

\section{New Courses}

CSHP and AdvancingIn are pleased to present the launch of a new eLearning platform called AdvancingIn 'Medication Management'. The following presentations from the CSHP 2010 Professional Practice Conference are now available. Please note that you will be required to register and log in to participate in these sessions (registration is free). To begin learning, visit www.cshp.ca under "Programs".

$\Rightarrow$ Aspirin for Primary Prevention: A Sober Second Look

$\Rightarrow$ Emerging Trends in Anticoagulation

$\Rightarrow$ A Paradigm Shift in the Treatment of Mood and Anxiety Disorders: What we know now about the role of Atypical Antipsychotics

$\Rightarrow$ The Light at the End of the Tunnel: Emerging Alternatives to Warfarin

\section{Additional Courses}

Visit the CSHP website for these additional online courses.

$\Rightarrow$ Canadian Diabetes Association Clinical Practice Guidelines: From Paper to Patient

$\Rightarrow$ Keeping Current with Medication Safety Research

$\Rightarrow$ The Bottom Line on Dyslipidemia Treatment for Pharmacists

CSHP... Supporting hospital pharmacists to excel and innovate in collaborative patient care. 